\title{
Outcome of patients with right heart thrombi: the Right Heart Thrombi European Registry
}

\author{
Marcin Koć ${ }^{\text {, Maciej Kostrubiec }}{ }^{1}$, Waldemar Elikowski ${ }^{2}$, Nicolas Meneveau ${ }^{3}$, \\ Mareike Lankeit ${ }^{4}$, Stefano Grifoni ${ }^{5}$, Agnieszka Kuch-Wocial ${ }^{6}$, Antoniu Petris ${ }^{7}$, \\ Beata Zaborska ${ }^{8}$, Branislav S. Stefanović ${ }^{9}$, Thomas Hugues ${ }^{10}$, Adam Torbicki ${ }^{11}$, \\ Stavros Konstantinides ${ }^{12}$ and Piotr Pruszczyk ${ }^{1}$ for the RiHTER Investigators
}

\begin{abstract}
Affiliations: ${ }^{1}$ Dept of Internal Medicine and Cardiology, The Medical University of Warsaw, Warsaw, Poland. ${ }^{2}$ Dept of Internal Medicine, J. Strus Hospital, Poznan, Poland. ${ }^{3}$ Dept of Cardiology, University Hospital of Besancon, Hospital Jean Minjoz, Besancon, EA 3920, France. ${ }^{4} \mathrm{Clinic}$ of Cardiology and Pneumology, Heart Center, University of Göttingen, Goettingen, Germany. ${ }^{5}$ Emergency Dept, AOU Careggi, Florence, Italy. ${ }^{6}$ Dept of Internal Medicine and Hypertension and Angiology, The Medical University of Warsaw, Warsaw, Poland. ${ }^{7}$ University of Medicine and Pharmacy Grigore T. Popa, Cardiology Clinic, Spitalul Clinic Judetean de Urgenta Sf. Spiridon, lasi, Romania. ${ }^{8}$ Dept of Cardiology, Postgraduate Medical School, Grochowski Hospital, Warsaw, Poland. ${ }^{9}$ Clinical Centre of Serbia, Cardiology Clinic, School of Medicine, University of Belgrade, Belgrade, Serbia. ${ }^{10}$ The Princess Grace Hospital Centre, Monaco. ${ }^{11}$ Dept of Pulmonary Circulation and Thromboembolic Diseases, Medical Center for Postgraduate Education, ECZ-Otwock, Otwock, Poland. ${ }^{12}$ Center for Thrombosis and Hemostasis, Gutenberg University of Mainz, Mainz, Germany.
\end{abstract}

Correspondence: Piotr Pruszczyk, Dept of Internal Medicine and Cardiology, The Medical University of Warsaw, Lindleya 4, 02-005 Warsaw, Poland. E-mail: piotr.pruszczykawum.edu.pl

ABSTRACT Our aim was the assessment of the prognostic significance of right heart thrombi (RiHT) and their characteristics in pulmonary embolism in relation to established prognostic factors.

138 patients (69 females) aged (mean \pm SD) $62 \pm 19$ years with RiHT were included into a multicenter registry. A control group of 276 patients without RiHT was created by propensity scoring from a cohort of 963 contemporary patients. The primary end-point was 30-day pulmonary embolism-related mortality; the secondary end-point included 30-day all-cause mortality. In RiHT patients, pulmonary embolism mortality was higher in 31 patients with systolic blood pressure $<90 \mathrm{mmHg}$ than in 107 normotensives ( $42 \%$ versus $12 \%, \mathrm{p}=0.0002$ ) and was higher in the 83 normotensives with right ventricular dysfunction (RVD) than in the 24 normotensives without RVD ( $16 \%$ versus $0 \%, \mathrm{p}=0.038)$. In multivariable analysis the simplified Pulmonary Embolism Severity Index predicted mortality (hazard ratio 2.43, 95\% CI 1.58-3.73; $\mathrm{p}<0.0001$ ), while RiHT characteristics did not. Patients with RiHT had higher pulmonary embolism mortality than controls ( $19 \%$ versus $8 \%, \mathrm{p}=0.003)$, especially normotensive patients with RVD ( $16 \%$ versus $7 \%, \mathrm{p}=0.02$ ).

30-day mortality in patients with RiHT is related to haemodynamic consequences of pulmonary embolism and not to RiHT characteristics. However, patients with RiHT and pulmonary embolism resulting in RVD seem to have worse prognosis than propensity score-matched controls.

@ERSpublications

Prognosis in patients with PE and RiHT is related to haemodynamic effects of PE, not RiHT morphology http://ow.ly/UCpja

Editorial comment in: Eur Respir J 2016; 47: 702-703 [DOI: 10.1183/13993003.01968-2015].

Received: May 242015 | Accepted after revision: Nov 062015 | First published online: Jan 212016

Conflict of interest: Disclosures can be found alongside the online version of this article at erj.ersjournals.com

Copyright @ERS 2016 


\section{Introduction}

Right heart thrombi (RiHT) can be detected echocardiographically in $\sim 4 \%$ of pulmonary embolism patients [1]. Although RiHT confirm pulmonary embolism, optimal therapy has not been defined and treatment selection is still a subject of debate. Most data are based on short case-series, their meta-analyses [2] or results of registries primarily focused on different aspects of pulmonary embolism [1, 3]. Good outcome of RiHT patients has been reported in some series [4-6], but in other reports mortality exceeded $20 \%$, even despite urgent thrombolysis $[1,7,8]$. RiHT morphology was reported to influence the prognosis and mobile thrombi were suggested to increase early mortality [9]. Although a meta-analysis of 177 cases suggested that thrombolytic therapy was associated with an improved survival when compared with either anticoagulation or surgery, the potential benefits of thrombolysis in stable patients with RiHT remain controversial [3]. The current European guidelines on pulmonary embolism management underline that, although the impact of RiHT on mortality in pulmonary embolism patients is yet unclear, the presence of RiHT should be considered a potentially life-threatening condition [10]. A multicenter, international Right Heart Thrombi European Registry (RiHTER) has been organised in order to assess the prognostic significance of RiHT characteristics according to their size and mobility, especially in relation to the established prognostic factors for 30-day mortality: haemodynamic instability, right ventricular dysfunction (RVD), simplified Pulmonary Embolism Severity Index (sPESI) score, and laboratory markers of myocardial injury and overload. Moreover, we assessed the potential prognostic value of RiHT and their potential influence on treatment selection in comparison with propensity score-matched (PSM) pulmonary embolism patients contemporarily managed in the centres participating in the registry.

\section{Methods}

Study design

17 centres from eight European countries (see Acknowledgements section) participated in an internet-based registry which collected anonymised clinical, echocardiographic and laboratory data of consecutive patients with RiHT and pulmonary embolism managed in these centres between January 2011 and February 2014. The following parameters were recorded: systolic blood pressure (sBP), heart rate, use of intravenous vasopressors at RiHT diagnosis, comorbidities (congestive heart failure, chronic obstructive pulmonary disease, neoplasms), and maximal plasma troponin and brain natriuretic peptide (BNP) levels. Pulmonary embolism was objectively confirmed in all haemodynamically stable patients. Only haemodynamically unstable patients with RiHT and RVD at echocardiography in whom objective pulmonary embolism diagnosis was not possible due to severe haemodynamic compromise could be included into the registry. Therapeutic interventions were evaluated. Hemodynamic instability was defined according the European Society of Cardiology (ESC) as: sBP $<90 \mathrm{mmHg}$ or a need for vasopressors without other coexisting causes. Shock index (SI) was defined as heart rate divided by sBP. The sPESI was defined as previously described [11].

Echocardiographic examinations were uploaded and were centrally re-evaluated. The following indices were assessed: dimensions of the right and left ventricles in the four-chamber apical view, McConnell's sign [12], tricuspid valve peak systolic gradient, and flattening of the interventricular septum. RiHTs were divided according to shape (snake-like, oval, perimural), mobility (mobile, immobile) and size in the largest dimension: $(\geqslant 5,<5 \mathrm{~cm})$ [9]. For RVD diagnosis we used the following echocardiographic and biochemical criteria: right ventricular/left ventricular $(\mathrm{RV} / \mathrm{LV})$ ratio $\geqslant 0.9$ in the four-chamber view, presence of McConnell's sign or intraventricular septum flattening; elevation of plasma BNP $\left(>90 \mathrm{pg} \cdot \mathrm{mL}^{-1}\right)$ or $\mathrm{N}$-terminal pro-BNP $\left(>500 \mathrm{pg} \cdot \mathrm{mL}^{-1}\right)$ or when plasma troponin T or troponin I levels exceeded $0.1 \mathrm{ng} \cdot \mathrm{mL}^{-1}$ or high-sensitive troponin levels were above the laboratory-specific upper reference limit.

Patients who were haemodynamically unstable at the time of RiHT diagnosis formed the high-risk pulmonary embolism (HRPE) group. Intermediate-risk pulmonary embolism (IRPE) was diagnosed when patients with $\mathrm{sBP} \geqslant 90 \mathrm{mmHg}$ presented with RVD and/or biochemical signs of myocardial injury. Low-risk pulmonary embolism (LRPE) comprised subjects who did not fulfil the criteria for HRPE or IRPE.

The study was prospective, but retrospective cases were also allowed. However, patients were considered to be eligible for analysis only when RiHT characteristics and right ventricular function were evaluated, clinical data allowed risk stratification according ESC criteria, and the assessment of sPESI score, and when data on applied therapy and 30-day outcome were available.

Additionally, a control group without RiHT was created by propensity score analysis from a cohort of 963 contemporary pulmonary embolism patients managed in the participating centres at the time of the registry. Propensity score matching was used to reduce possible inherent bias between patients with and without RiHT. We performed a 1:2 matched analysis on the basis of the estimated propensity score of each patient. Finally, a control group without RiHT comprised 276 propensity score-matched (PSM) pulmonary embolism patients. 


\section{End-points}

The primary end-point was 30-day pulmonary embolism-related mortality in relation to the clinical severity of pulmonary embolism and RiHT characteristics. The secondary end-points included 30-day all-cause mortality in relation to the clinical severity of pulmonary embolism and RiHT characteristics, and a comparison of 30-day pulmonary embolism-related mortality between pulmonary embolism patients with RiHT and PSM controls without RiHT. Pulmonary embolism-related mortality was diagnosed when haemodynamic instability and/or shock preceded death, or when a patient experienced pulmonary embolism recurrence. When an evident alternative cause of death was reported, such as severe bleeding, neoplasm or sepsis with no data for pulmonary embolism-related death, a nonpulmonary embolism death was diagnosed and this contributed to 30-day all-cause mortality.

\section{Statistical analysis}

Data with a normal distribution are expressed as mean \pm SD. Parameters without such a distribution are expressed as median (range). t- or Mann-Whitney tests were used for comparisons between two groups, and for more than two groups we used ANOVA or Kruskal-Wallis tests. Fisher's exact or Chi-squared tests were used to compare discrete variables. Sensitivity, specificity, positive predictive value (PPV) and negative predictive value (NPV) were calculated. The impact of the analysed parameters on 30-day survival was evaluated using univariable and multivariable Cox proportional hazards regression. Kaplan-Meier analysis was used to investigate cumulative 30-day survival. All tests were two-sided. Analyses were considered significant at $\mathrm{p}<0.05$. We compared prospective and retrospective cases with respect to clinical and RiHT characteristics, treatment and outcome.

Propensity score analysis was used to select a control group without RiHT from a cohort of 963 contemporary patients managed in participating centres at the time of the registry. With the use of a multivariable logistic regression model that included basic risk parameters as the independent variables, the probability of a patient being assigned to the group with RiHT was determined. The goodness-of-fit of the propensity score model was obtained by c statistics, a measure of the discriminatory power of a predictive model. The variables included in the propensity score model were age, sex, RV/LV ratio, heart rate, comorbidities and assignment to risk group according to ESC guidelines. We performed a 1:2 matched analysis on the basis of the estimated propensity score of each patient. A control group without RiHT comprised 276 PSM pulmonary embolism patients.

STATISTICA version 10 (StatSoft, Tulsa, OK, USA) and MedCalc (MedCalc Software, Ostend, Belgium) were used for calculations. The RiHTER protocol was approved centrally by the Ethics Committee at the Medical University of Warsaw and locally wherever required. Although adverse events were not adjudicated by an independent committee, they were centrally evaluated by three investigators and the final decision was reached by consensus.

\section{Results}

\section{Clinical characteristics of patients}

Data of 158 patients with RiHT were incorporated into the database. 138 of them were eligible for the analysis (69 females, aged $62 \pm 19$ years), while 20 other cases were excluded from the analysis due to lack of significant data regarding clinical presentation, RiHT characteristics, treatment or outcome. 62 subjects underwent echocardiography due to suspected pulmonary embolism and in 63 patients it was performed after pulmonary embolism diagnosis. In the remaining 13 patients RiHT was an incidental finding. Pulmonary embolism was objectively confirmed in all haemodynamically stable patients. In 31 patients haemodynamic instability was present at the time of RiHT diagnosis (HRPE), 25 of them received intravenous vasopressors intravenously and seven subjects were under cardiopulmonary resuscitation. In four subjects RiHT and severe RVD were diagnosed during unsuccessful cardiopulmonary resuscitation and subsequent objective pulmonary embolism diagnosis was not possible. 107 stable patients formed the non-HRPE (nHRPE) group. LRPE was diagnosed in 24 of them, while 83 patients with RVD formed the IRPE group (table 1). The RiHTER population comprised 72 prospective and 66 retrospectively enrolled cases; however, we found no differences in clinical parameters and pulmonary embolism severity between these two groups. A control group included 276 pulmonary embolism patients without RiHT matched with patients with RiHT in a 2:1 ratio according to the individual propensity score.

\section{Characteristics of RiHT}

RiHT were divided according to their shape (snake-like: 81 cases; oval: 44 cases; perimural: 13 cases), mobility (mobile: 118 cases; immobile: 20 cases), and size ( $\geqslant 5 \mathrm{~cm}: 40$ cases; $<5 \mathrm{~cm}$ : 98 cases); the smallest $(8 \times 4 \mathrm{~mm})$ was found in an IRPE patient, while the largest $(250 \times 25 \mathrm{~mm})$ was in another IRPE patient. There were no differences in RiHT size, shape and mobility between unstable and stable patients or between IRPE and LRPE (table 1). 
TABLE 1 Clinical characteristics of patients with right heart thrombi (RiHT), control cohort and propensity score-matched (PSM) controls

\begin{tabular}{|c|c|c|c|c|c|c|c|c|c|}
\hline & $\begin{array}{l}\text { Control } \\
\text { cohort }\end{array}$ & $\begin{array}{l}\text { PSM } \\
\text { controls }\end{array}$ & All RiHT & HRPE & $\begin{array}{l}\mathrm{p} \text {-value (HRPE } \\
\text { versus nHRPE) }\end{array}$ & nHRPE & IRPE & $\begin{array}{l}\text { p-value (IRPE } \\
\text { versus LRPE) }\end{array}$ & LRPE \\
\hline Subjects & 963 & 276 & 138 & 31 & & 107 & 83 & & 24 \\
\hline Age years & $64 \pm 18$ & $65 \pm 17$ & $62 \pm 19$ & $63 \pm 21$ & 0.87 & $61 \pm 19$ & $62 \pm 20$ & 0.23 & $58 \pm 18$ \\
\hline Female/male & $542 / 421$ & $126 / 150$ & $69 / 69$ & $17 / 14$ & 0.54 & $52 / 55$ & $38 / 45$ & 0.28 & $14 / 10$ \\
\hline $\mathrm{sBP} \mathrm{mmHg}$ & $127 \pm 24$ & $124 \pm 26$ & $109 \pm 25$ & $74 \pm 12$ & $<0.0001$ & $119 \pm 19$ & $118 \pm 19$ & 0.61 & $120 \pm 18$ \\
\hline Heart rate bpm & $91 \pm 21$ & $102 \pm 23$ & $103 \pm 22$ & $117 \pm 18$ & $<0.05$ & $102 \pm 22$ & $102 \pm 22$ & 0.01 & $92 \pm 19$ \\
\hline $\mathrm{SaO}_{2} \%$ & $93 \pm 11$ & $92 \pm 7$ & $91 \pm 6$ & $86 \pm 9$ & 0.02 & $91 \pm 6$ & $89 \pm 16$ & 0.50 & $91 \pm 7$ \\
\hline Comorbidities ${ }^{\#}$ & $176(18)$ & $65(24)$ & 76 (55) & $16(51)$ & 0.66 & $60(56)$ & $44(53)$ & 0.34 & $16(66)$ \\
\hline Shock index & $0.75 \pm 0.29$ & $0.87 \pm 0.33$ & $1.00 \pm 0.2$ & $1.59 \pm 0.39$ & $<0.0001$ & $0.86 \pm 0.2$ & $0.88 \pm 0.24$ & 0.01 & $0.74 \pm 0.21$ \\
\hline sPESI score $\geqslant 1 / 0$ & $600 / 363$ & $198 / 78$ & $122 / 16$ & $31 / 0$ & $<0.05$ & $91 / 16$ & $73 / 10$ & 0.21 & $18 / 6$ \\
\hline sPESI score & $1.06 \pm 1.08$ & $1.4 \pm 1.2$ & $1.4 \pm 0.9$ & $3.0 \pm 0.9$ & $<0.0001$ & $1.4 \pm 0.9$ & $1.4 \pm 0.9$ & 0.24 & $1.2 \pm 1.0$ \\
\hline RiHT shape S/0/P & & & $81 / 44 / 13$ & $22 / 7 / 2$ & 0.22 & $59 / 37 / 11$ & $47 / 26 / 10$ & 0.21 & $12 / 11 / 1$ \\
\hline RiHT mobility M/I & & & $118 / 20$ & $30 / 1$ & 0.08 & $88 / 19$ & $66 / 17$ & 0.29 & $22 / 2$ \\
\hline RiHT size $\leqslant 5 />5 \mathrm{~cm}$ & & & $98 / 40$ & $20 / 11$ & 0.37 & $78 / 29$ & $60 / 23$ & 0.99 & $18 / 6$ \\
\hline RiHT protruding into PFO & & & $14(10)$ & $3(10)$ & 0.81 & $11(10)$ & $9(11)$ & 0.98 & $2(8)$ \\
\hline
\end{tabular}

Data are presented as $n$, mean $\pm S D$ or $n(\%)$, unless otherwise stated. HRPE: high-risk pulmonary embolism; nHRPE: non-HRPE; IRPE: intermediate-risk pulmonary embolism; LRPE: low-risk pulmonary embolism; sBP: systolic blood pressure; SaO ${ }_{2}$ : arterial oxygen saturation; sPESI: simplified Pulmonary Embolism Severity Index; S: snake-like; O: oval; P: perimural; M: mobile; I: immobile; PFO: patent foramen ovale. \#: congestive heart failure, chronic obstructive pulmonary disease, neoplasms.

\section{Treatment and clinical course}

Thrombolysis in the RiHT group was used in $56(41 \%)$ patients. Interventional therapy was the first therapeutic choice in 15 (11\%) patients (surgery 14 in patients, percutaneous RiHT removal in one patient). The remaining 67 (48\%) patients were anticoagulated only (table 2 ). Thrombolysis was more frequently used in HRPE patients than in nHRPE patients $(61 \%$ versus $35 \%, \mathrm{p}<0.01)$. Although there were no statistical differences in type of treatment between LRPE and IRPE patients, thrombolysis was used almost twice as often in patients with signs of RVD (39\% and 21\%, not significant). Pulmonary embolism-related deaths occurred in 26 cases (30-day pulmonary embolism-related mortality 19\%). There was a difference in pulmonary embolism-related mortality between HRPE and nHRPE patients ( $42 \%$ versus $12 \%, \mathrm{p}=0.0002$ ) (table 2) and also in stable patients according to the presence of RVD: IRPE versus LRPE patients (16\% versus $0 \%, \mathrm{p}=0.038$ ). The majority of deaths $(62 \%)$ occurred within the first $24 \mathrm{~h}$ (figure 1 ).

Eight causes of nonpulmonary embolism-related deaths included advanced neoplasms (three cases), left ventricular failure with severe left ventricular systolic dysfunction (two cases), sepsis (one case), intracranial haemorrhage (two cases) and acute kidney injury (one case); all-cause mortality reached $25 \%$.

Among 31 HRPE patients, seven subjects were under cardiopulmonary resuscitation at the time of RiHT diagnosis and four of them died. We found no differences in mortality in haemodynamically unstable RiHT patients according to the applied therapy. nHRPE was diagnosed in 107 patients. This group included 24 subjects with LRPE and 83 patients with IRPE. Thrombolysis was used in 35\% of non-HRPE patients, anticoagulation in 55\% and invasive treatment in the remaining $10 \%$. All 16 pulmonary

TABLE 2 Treatment and outcome according to the clinical characteristics of 138 patients with right heart thrombi

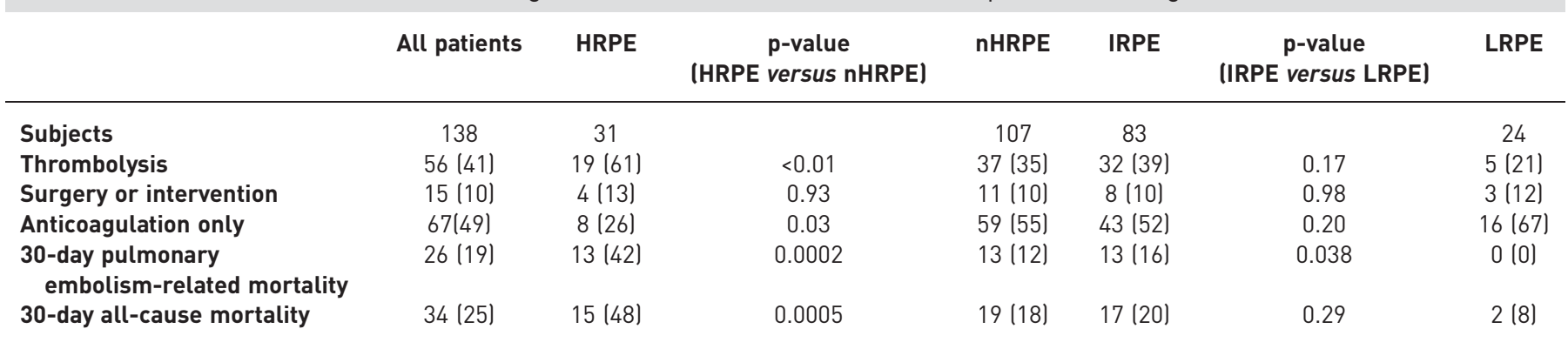

Data are presented as $\mathrm{n}$ or $\mathrm{n}(\%)$, unless otherwise stated. HRPE: high-risk pulmonary embolism; nHRPE: non-HRPE; IRPE: intermediate-risk pulmonary embolism; LRPE: low-risk pulmonary embolism. 

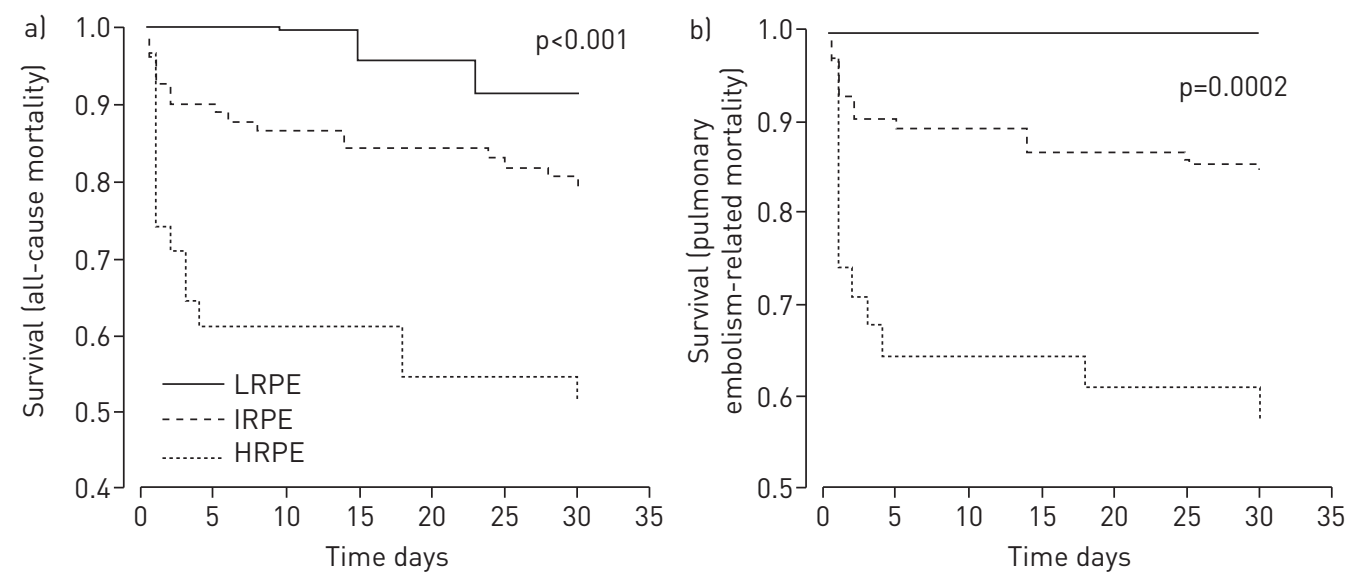

FIGURE 1 Kaplan-Meier survival curves in 138 patients with right heart thrombi according to pulmonary embolism severity defined by the European Society of Cardiology: al all-cause mortality and b) pulmonary embolism-related mortality. LRPE: low-risk pulmonary embolism; IRPE: intermediate-risk pulmonary embolism; HRPE: high-risk pulmonary embolism.

embolism-related deaths in nHRPE patients occurred in the subgroup with IRPE, while no pulmonary embolism-related deaths occurred in LRPE patients. We observed significant differences in pulmonary embolism-related mortality between the IRPE and LRPE groups ( $16 \%$ versus $0 \%, \mathrm{p}=0.038)$. Patients with RiHT had higher 30-day pulmonary embolism-related mortality than PSM controls (19\% versus $8 \%$ $\mathrm{p}=0.003$ ) (figure 2). This effect was present in the IRPE group ( $16 \%$ versus $7 \%, \mathrm{p}=0.02)$, while in the HRPE group we observed a trend only $(42 \%$ versus $22 \% \mathrm{p}=0.07)$. There were no pulmonary embolism-related deaths in LRPE groups with or without RiHT. We found that in the whole RiHT group aggressive treatment (thrombolysis or embolectomy) was more frequently used than in PSM controls ( $51 \%$ versus $10 \%, \mathrm{p}<0.001)$.

\section{Mortality predictors}

Univariable analysis showed that sPESI score, SI and sBP $<90 \mathrm{mmHg}$, but not RiHT parameters, were predictors of pulmonary embolism-related and all-cause mortality in the whole RiHT group (table 3). However, in multivariable analysis only sPESI score predicted 30-day all-cause and pulmonary embolism-related mortality (hazard ratio 2.56, 95\% CI 1.71-3.84; $\mathrm{p}<0.001$ and hazard ratio 2.43 , 95\% CI $1.58-3.73 ; \mathrm{p}<0.0001$, respectively).

In the group with nHRPE, sPESI and SI were also univariate predictors for both all-cause and pulmonary embolism-related mortality (table 3). However, in multivariable analysis only SI predicted 30-day all-cause and pulmonary embolism-related mortality (hazard ratio 29.8 , 95\% CI 3.3-271.1; $\mathrm{p}=0.003$ and hazard ratio 83.99, 95\% CI 5.5-1295.7; p=0.002, respectively). Importantly, none of 24 low-risk patients (17.4\% of all patients) died due to pulmonary embolism (NPV 100\%). Moreover, there were no deaths in the group with an sPESI of 0 , which included 16 patients.

FIGURE 2 30-day pulmonary embolism-related mortality in 138 patients with right heart thrombi $(\mathrm{RiHT}(+))$ and 276 propensity score-matched controls (RiHT(-)). HRPE: high-risk pulmonary embolism; IRPE: intermediate-risk pulmonary embolism; LRPE: low-risk pulmonary embolism.

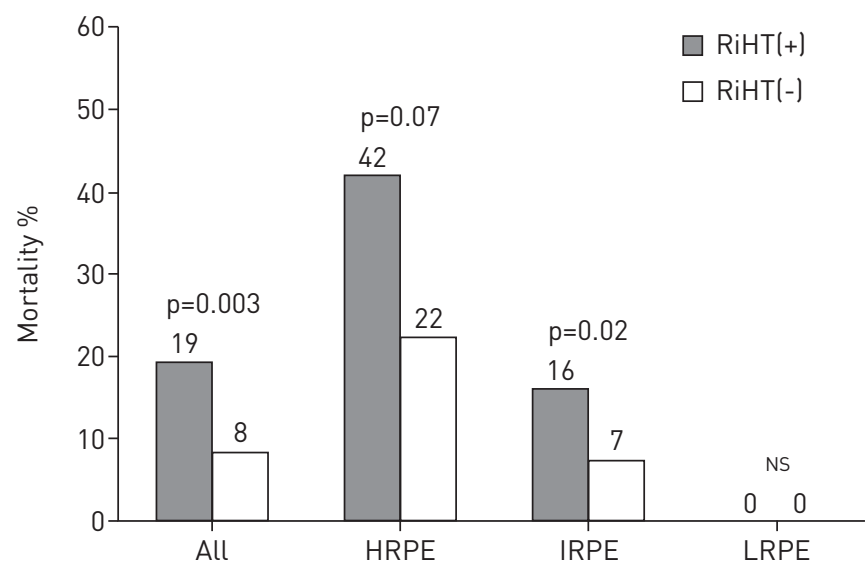


TABLE 3 Univariable 30-day mortality predictors in patients with right heart thrombi

\begin{tabular}{|c|c|c|c|c|}
\hline & \multicolumn{2}{|c|}{ All-cause mortality } & \multicolumn{2}{|c|}{$\begin{array}{c}\text { Pulmonary } \\
\text { embolism-related mortality }\end{array}$} \\
\hline & Hazard ratio $(95 \% \mathrm{CI})$ & p-value & Hazard ratio $(95 \% \mathrm{CI})$ & p-value \\
\hline \multicolumn{5}{|l|}{ All $n=138$} \\
\hline sPESI score & $2.56(1.71-3.84)$ & $<0.001$ & 2.43 (1.58-3.73) & $<0.0001$ \\
\hline Shock index & $5.82(2.07-16.41)$ & 0.001 & 8.96 (2.82-28.48) & 0.0002 \\
\hline $\mathrm{sBP}<90 \mathrm{mmHg}$ & 4.89 (2.07-11.54) & $<0.001$ & 5.18 (2.01-13.39) & 0.001 \\
\hline \multicolumn{5}{|l|}{ nHRPE n=107 } \\
\hline sPESI score & $2.41(1.42-4.07)$ & 0.001 & $1.94(1.11-3.40)$ & 0.02 \\
\hline Shock index & $29.8(3.3-271.1)$ & 0.003 & 83.99 (5.5-1295.7) & 0.002 \\
\hline
\end{tabular}

sPESI: simplified Pulmonary Embolism Severity Index; sBP: systolic blood pressure; nHRPE: non-high-risk pulmonary embolism.

\section{Discussion}

RiHT are still regarded as a significant predictor of poor prognosis in pulmonary embolism $[1,10]$. However, there are also data indicating that RiHT are only "in transit" thrombi which should be considered as a confirmation of venous thromboembolism and prognosis is predominantly related to the haemodynamic status rather than the RiHT [3]. Using propensity score matching, we showed that pulmonary embolism-related mortality was higher in the whole RiHT group when compared with PSM controls (19\% versus $8 \%, \mathrm{p}=0.003$ ). This was mostly due to the mortality difference in the IRPE group (16\% versus $7 \%$, $\mathrm{p}=0.02$ ), while in the HRPE group we observed a trend only ( $42 \%$ versus $22 \%, \mathrm{p}=0.07)$. We observed a significant difference in pulmonary embolism-related mortality in the RiHT group according to pulmonary embolism severity and it was significantly lower in the nHRPE group when compared with unstable patients, and between LRPE and IRPE patients ( $0 \%$ versus $16 \%, \mathrm{p}=0.038)$. Therefore, haemodynamic instability strongly indicated a mortality risk. Importantly, the majority of deaths (62\%) occurred within the first $24 \mathrm{~h}$ after diagnosis. In the whole studied group, univariable analysis showed that sPESI score, SI and sBP $<90 \mathrm{mmHg}$, but not RiHT parameters, were predictors of pulmonary embolism-related and all-cause mortalities. Moreover, in the multivariable analysis, only sPESI score predicted 30-day all-cause and pulmonary embolism-related mortality. Importantly, none of the 24 haemodynamically stable patients with preserved right ventricular function (17.4\% of all patients) died due to pulmonary embolism (NPV $100 \%)$. sPESI of 0 also identified 16 patients with a good 30-day prognosis despite the presence of RiHT.

The all-cause mortality at 30 days was similar to that reported of $21 \%$ in a subgroup of the International Cooperative Pulmonary Embolism Registry population with pulmonary embolism and coexisting RiHT [1]. This mortality rate in patients with RiHT is also much higher than recently reported (2.4-3.2\%) in similarly defined IRPE patients enrolled into a large randomised controlled trial regardless of the presence of RiHT [13]. Our data suggest an increased mortality risk of patients with RiHT and RVD. However, in patients with LRPE we observed no pulmonary embolism-related mortality. Importantly, we found no differences in RiHT morphology and function between survivors and nonsurvivors.

Since there are no precise data on the prognosis of patients with RiHT, their proper management is still a matter of debate, especially in patients haemodynamically stable on admission in whom anticoagulation is the treatment of choice according to current guidelines. We found that in the whole RiHT group aggressive treatment (thrombolysis or embolectomy) was significantly more frequently used than in PSM controls ( $51 \%$ versus $10 \%, \mathrm{p}<0.0001$ ). This difference was also present in the LRPE ( $33 \%$ versus $3 \%$, $\mathrm{p}<0.001)$ and IRPE $(51 \%$ versus $5 \%, \mathrm{p}<0.001)$ groups, while it was not significant in the HRPE group $(74 \%$ versus $56 \%, \mathrm{p}=0.11$ ). However, we were not able to demonstrate any benefit of aggressive therapy in the RiHTER population.

\section{Study limitations}

The main limitation of RiHTER is the lack of standardised criteria for management of pulmonary embolism patients, which makes comparison between efficacy and safety of different treatments difficult. RiHTER included a relatively limited number of patients. Although pulmonary embolism was objectively confirmed in all controls, it was not confirmed in four RiHT patients in whom RiHT and RVD were visualised during unsuccessful cardiopulmonary resuscitation. We tried to maintain a high quality of data. Therefore, echocardiographic examinations were uploaded and centrally re-evaluated. However, causes of deaths were not adjudicated by an independent committee. The study was prospective, but retrospective 
cases could also be enrolled. However, pre-defined echocardiographic, laboratory and clinical parameters were available in all cases. Importantly, we found no significant differences in clinical characteristics, RiHT characteristics or applied treatment and outcome between prospective and retrospective patients.

\section{Conclusion and clinical implications}

We found that the currently recommended methods of prognostic stratification in acute pulmonary embolism are also applicable to patients with $\mathrm{RiHT}$, and their outcome was predominantly related to the haemodynamic and clinical consequences of acute pulmonary embolism itself, and not to morphology of RiHT. However, our results indicate that 30 -day pulmonary embolism-related mortality in patients with RiHT is increased when compared with PSM controls. Patients with RiHT and pulmonary embolism resulting in RVD seem especially to have a worse prognosis than PSM controls.

\section{Acknowledgements}

List of co-authors/RiHTER Investigators with number of included patients: Waldemar Elikowski, Dept of Internal Medicine, J. Strus Hospital, Poznan, Poland (38 patients); Nicolas Meneveau, Dept of Cardiology, University Hospital of Besancon - Hospital Jean Minjoz, Besancon, France (17 patients); Antoniu Petris, University of Medicine and Pharmacy Grigore T. Popa, Cardiology Clinic, Spitalul Clinic Judetean de Urgenta Sf. Spiridon, Iasi, Gabriel Tatu-Chitoiu, Emergency Hospital Bucharest, Calin Pop, County Emergency Hospital Baia Mare, University "Vasile Goldis", Arad, Romania (16 patients); Marcin Koć (Study Coordinator), Maciej Kostrubiec and Piotr Pruszczyk, Dept of Internal Medicine and Cardiology, The Medical University of Warsaw, Poland (15 patients); Mareike Lankeit, Dept of Cardiology, Heart Centre, University of Goettingen, Goettingen, Germany (13 patients); Stefano Grifoni, Emergency Dept, AOU Careggi, Florence, Italy (eight patients); Beata Zaborska, Dept of Cardiology, Postgraduate Medical School, Grochowski Hospital, Warsaw, Poland (six patients); Agnieszka Kuch-Wocial, Dept of Internal Medicine Hypertension and Angiology, The Medical University of Warsaw, Poland (six patients); Branislav S. Stefanović, Cardiology Clinic, Clinical Centre of Serbia, School of Medicine, University of Belgrade, Belgrade, Serbia (four patients); Thomas Hugues The Princess Grace Hospital Centre, Monaco (four patients); Bożena Sobkowicz, Cardiology Dept, Medical University of Bialystok, Poland (three patients); Marek Postuła, Cardiology Dept, Central Clinical Hospital of the Ministry of Interior, Warsaw, Poland (two patients); Anna Klisiewicz, Dept of Congenital Cardiac Defects, Institute of Cardiology, Warsaw, Poland (two patients); Cecilia Becattini, Internal and Cardiovascular Medicine and Stroke Unit, University of Perugia, Italy (two patients); Paweł Grzelakowski, Cardiology and Cardiosurgery Dept, 10th Military Hospital, Bydgoszcz, Poland (one patient); Andrzej Tomaszewski, Cardiology Dept, Medical University of Lublin, Poland (one patient); Adam Torbicki, Dept of Pulmonary Circulation and Thromboembolic Diseases, Medical Center for Postgraduate Education, ECZ-Otwock, Otwock, Poland; Stavros Konstantinides, Center for Thrombosis and Hemostasis, Gutenberg University of Mainz, Mainz, Germany.

\section{References}

1 Torbicki A, Galie N, Covezzoli A, et al. Right heart thrombi in pulmonary embolism: results from the International Cooperative Pulmonary Embolism Registry. J Am Coll Cardiol 2003; 41: 2245-2251.

2 Rose PS, Punjabi NM, Pearse DB. Treatment of right heart thromboemboli. Chest 2002; 121: 806-814.

3 Casazza F, Becattini C, Guglielmelli E, et al. Prognostic significance of free-floating right heart thromboemboli in acute pulmonary embolism: results from the Italian Pulmonary Embolism Registry. Thromb Haemost 2014; 111: 53-57.

4 Ferrari E, Benhamou M, Berthier F, et al. Mobile thrombi of the right heart in pulmonary embolism: delayed disappearance after thrombolytic treatment. Chest 2005; 127: 1051-1053.

5 Pierre-Justin G, Pierard LA. Management of mobile right heart thrombi: a prospective series. Int J Cardiol 2005; 99: $381-388$

6 Casazza F, Bongarzoni A, Centonze F, et al. Prevalence and prognostic significance of right-sided cardiac mobile thrombi in acute massive pulmonary embolism. Am J Cardiol 1997; 79: 1433-1435.

7 Chartier L, Bera J, Delomez M, et al. Free-floating thrombi in the right heart: diagnosis, management, and prognostic indexes in 38 consecutive patients. Circulation 1999; 99: 2779-2783.

8 Kinney EL, Wright RJ. Efficacy of treatment of patients with echocardiographically detected right-sided heart thrombi: a meta-analysis. Am Heart J 1989; 118: 569-573.

9 European Working Group on Echocardiography. The European Cooperative Study on the clinical significance of right heart thrombi. Eur Heart J 1989; 10: 1046-1059.

10 Konstantinides S, Torbicki A, Agnelli G, et al. 2014 ESC Guidelines on the diagnosis and management of acute pulmonary embolism. The Task Force for the Diagnosis and Management of Acute Pulmonary Embolism of the European Society of Cardiology (ESC). Endorsed by the European Respiratory Society (ERS). Eur Heart J 2014; 35: 3033-3069.

11 Jimenez D, Aujesky D, Moores L, et al. Simplification of the pulmonary embolism severity index for prognostication in patients with acute symptomatic pulmonary embolism. Arch Intern Med 2010; 170: 1383-1389.

12 McConnell MV, Solomon SD, Rayan ME, et al. Regional right ventricular dysfunction detected by echocardiography in acute pulmonary embolism. Am J Cardiol 1996; 78: 469-473.

13 Meyer G, Vicaut E, Danays T, et al. Fibrinolysis for patients with intermediate-risk pulmonary embolism. $N$ Engl $J$ Med 2014; 370: 1402-1411. 